\title{
Geoliteracy, cartology, and classroom mobile projects or game
}

\author{
Yaïves Ferland $^{\text {a, } * \text {, Margot Kaszap }}{ }^{\text {b }}$ \\ ${ }^{a}$ Université Laval, Québec, Canada, yaives.ferland@scg.ulaval.ca \\ ${ }^{b}$ Université Laval, Québec, Canada, margot.kaszap@fse.ulaval.ca \\ * Corresponding author
}

Keywords: Geographic literacy, Cartology, Experiential learning, Learning threshold, Mobile serious game, trajectories, mobile activity

\begin{abstract}
:
Reading and drawing maps were always part of the curriculum in most countries at primary school levels. Even so, numerous adults seem unable to find their way when using a map or changing a travel direction toward a distant place. They are geographically illiterate and inefficient, which implies a societal concern even if today they can access to some technological devices to help. People get lost following the device without analysing the landscape to realise that they are going in a wrong place.

Geographic literacy, or "geoliteracy," can be defined as a set of stabilized and adaptive cognitive abilities and functional competencies to handle, by self, geographical realities and cartographical representations. According to Edelson (2012), the three components of an achieved geoliteracy are to develop consciousness of geographical interactions (understanding of human and natural systems in space and places), interconnections (geographic reasoning on structures and relationships), and implications (systematic planning and decision-making). The concept of geoliteracy, developed over the past decades (Ball, 2003), implies that one has to work on methodologies that will help children to develop these map skills at elementary school (Catling, 1996). The challenge is about improving their geographic literacy and fluency, and reciprocally their map-reading and map-using competencies called "cartology," before the turning point of their adolescence, a critical "threshold" of their cognitive development. A definition of cartology could be "to make the map talking," even for telling an improvised and adapted story from clues that the mapmaker did not explicitly draw on it. The point is not just about a cognitive process of deduction or abduction, but spatial or geographical reasoning.
\end{abstract}

Our first research interest went toward the development of an educative serious game (Kaufman \& Sauvé, 2010) on mobile devices (Kaszap, Stan \& Ferland, 2015) for letting elementary school pupils (i.e. primary school students) playing actively outdoors while gathering pictures and data in order to learn geographical facts, concepts, patterns and processes at the local level of their place, at least. For this, we didactically designed a methodological framework with maps and other components of an educative game, following an increasing complex structure of scenarios, trajectories, and themes. To do so, we base this framework on the four cognitive development stages for geospatial representation by children (Piaget, 1967), the experiential learning cycle model (Kolb, 1978, 1984) and the carto-graphical semiology (Bertin, 1967) of visual variables, associated with the successive stages of map-making, visualization, and cartology (Ferland, 2018).

One does no more reserve such a framework capacity to traditional neither electronic views of a competitive "game" with scores, goals, and prizes. It goes far beyond both passive video game and occasional geocaching/orienteering parties, engaging the child to play to fieldwork and map making. The presentation will explain and discuss the conceptual and methodological structure of this geoliteracy experiential framework (Figure 1) with a composite example of activities settled in scenarios of data collection in the vicinity of the school.

The proposed methodological framework must be viewed as a complete developmental K-12 ${ }^{1}$ program. It conveniently starts from data collection on the terrain within a map-making process (K-6, up to age 12) that can be done at first with young children within the school surroundings. Later, older teenagers will come to explore complex environments by interpreting maps through an original cartologic process (7-12).

The main concern to address early at school shall no longer be "where are the roads, the buildings, etc.," but rather the fundamental questions in geography: "why are they there?" "why is this entity or phenomenon located at that place or on this site?" If the children's geospatial cognitive development was weak at school, then that impedes them from comprehending geographical concepts, structures, and information later as adults. If a student does not succeed in passing over a kind of learning 'threshold' (Anderson, 2003) by about age 12, the few abilities that this student has feebly acquired may vanish, leading to a lowered interest in map reading nor even usage at the secondary school level. Later, it will be very hard for the student to restart learning the same matter without the necessary mental frameworks to organize geographical concepts and relations into an actionable knowledge, while becoming an adult. When the student faces this geoliteracy challenge, the geographical map appears as the best, powerful, and necessary support or instrument of geospatial knowledge representation.

${ }^{1}$ K-12: from Kindergarten (age 4-5) to 12 grade (age 17-18). 
The ultimate pedagogical goal of our framework is to bring children through each step of a game, i.e. a structured set of pleasant activities that the class teacher prepares according to some curriculum themes of local interest. Within the recursive experiential cycle (Kolb, 1978, 1984), experiential learning goes normally through four phases crossed by four trends, which delimit four learning 'styles' in sequence: diverger, assimilator, converger, and accommodator (note that this structure is by no way deterministic, but progressive for any individual, with different triggers or starting points, at will). Among the specified activities along the experiential learning cycle, our geospatial geoliteracy model selects the following ones (Figure 1). First, after reflexive observation and collect of data on the terrain, one may perform some degree of analysis in order to elaborate an abstract synthesis that realize a map, which allows visualization and simulations on it. At that stage, a number of well-designed activities come to develop map-building skills of the students. Then, understanding how maps work, they will be able to engage in active experimentation by decision of some plan leading to a concrete experience, what requires better skills to read and interpret map for making sense from it, this being called 'cartology'. This kind of cycle supports easily Piaget's learning phases, from topologic perception to spatial conceptualisation, as well as the three main cartographic processes of map-making, reflexive visualization, and mapreading, which sustain any geographical reasoning.

The teacher prepares a sequence of geographical tasks to perform in a progressive set of activities, like a game or a project, with respect to the different learning styles, for exposing practically the pupils to the cartographical process of making a plan, then a map to be used thereafter by other ones. Students are eager to go outdoors on the terrain to gather data in order to answer a question on a theme of investigation related to some discipline principles of a curriculum objective at their education degree. Such themes are very diverse, from landscape features to ancient architecture or energy network. Sub-themes may refer to local industries or institutions, art and culture, circulation, geomorphology, sport, toponymy, and are proper to the rural, urban, suburban, maritime or touristic environment of the school or the neighbourhood.

Students will be asked to consider a designed scenario of typical steps (or 'rounds' of a game) that tells a progressive plot, a home place story that they will have to show on successive versions of a map that they draw themselves while taking field-notes on their way to answering significant orientation questions. This progression in complexity levels of scenario types may start with choosing between right or left to reach the next point of interest, then trying to plan both the shortest and/or the more pleasant path. The competency is here to find and analyse first-hand information needed, then to decide and progress to the next location spot of the story, while gathering new data. All the spots may be given, or only the first one to start. In level 2 scenario, two spots are given, at the start or the first and the final ones of the scenario. The player has to analyse the given problem to determine the best step to get the information required to know the following spot or path, or to locate the next pair of spots to select. In raising the complexity of the scenario, one develops competencies to compare, locate, orient, observe, and deduce (Kaszap, Stan \& Ferland, 2015; Klopfer, 2008). The other types of scenarios that we considered go up to level 6 of complexity, where resulting competencies are likely to critique, solve spatial problem, imagine situations, choice, and make a deeper sense of place.

They levy data by the means of photograph or sketch, monument or public building characteristics and attributes (e.g., street address), geographic information found by GPS or on the Web. Doing so, the teacher designs the 'rules' of the game, like an task sheet, with pedagogic objectives and goal of play, technology support tools, etc.

The scenarios are closely related to a spectrum of trajectory types going from place to place, in increasing complexity as the project, the game or the story proceeds toward a certain geographical knowledge (the 'goal' of the game). In fact, these types are based on geometrical primitives: point, succession of points, cluster of points, line, both sides of a line, crossroad, pole (central point), axis, vector (one way), polyline, polygon, network or lattice (street, bus, watershed, energy distribution), open surface, limited surface, relief surface, relief volume (mountain, high-density zone), and so on. Their purpose is to initiate firmly the children to complex spatial schemes, reaching a comprehension of second-order thinking. Another kind of trajectories refers to oriented (topological) and regulated ways to enter, exit, or forbid access in some dynamic urban structures where the primitives would be borders, gates, and spatial processes (Ferland \& Mercier, 2004).

Thereafter, students should come back in the classroom in order to compare their collected data and draw a plan or map from them, and then explain in conclusion what happened to the story (and what they learned) due to the spatial organisation of the site or area, in their town or on the field. Finally, the evaluation proceeds with their peers trying to get new information and to learn from the maps exchanged with each other. The pedagogical result encompasses both concrete display of a terrain (on paper or screen) and learned cognitive 'forms' in the mind. Only such mental or cognitive representations allow structuring, interpreting, and recalling on demand from memory geospatial information on location, distance, or orientation, within a situation that occurs at geographical scales, viz. beyond the horizon. Being cautious, one cannot presume that apparent forms shown on the map replicate in the psychological representation in the student's mind.

At this point, only the first half of the experiential learning cycle is completed and the cognitive development process is achieved just at the phase associated to the threshold of operational comprehension. Now, the students know how to describe a spatial situation and make a map; this is good but not enough. The challenge remains to learn from this quite technical knowledge how to read a map deeply, any map, and to get dense information from it; it is a reflexive, analytical, abstract new phase called visualization. That phase engages a second process along the second half of the experiential learning cycle, which mirrors or complements the cartographic one: a cartological process. However, since it would imply a hypothetical fifth stage of cognitive development beyond Piaget's theory, we consider that cartology corresponds preferably to teenagers in secondary school degrees. Methodological and experimental developments are in progress. 


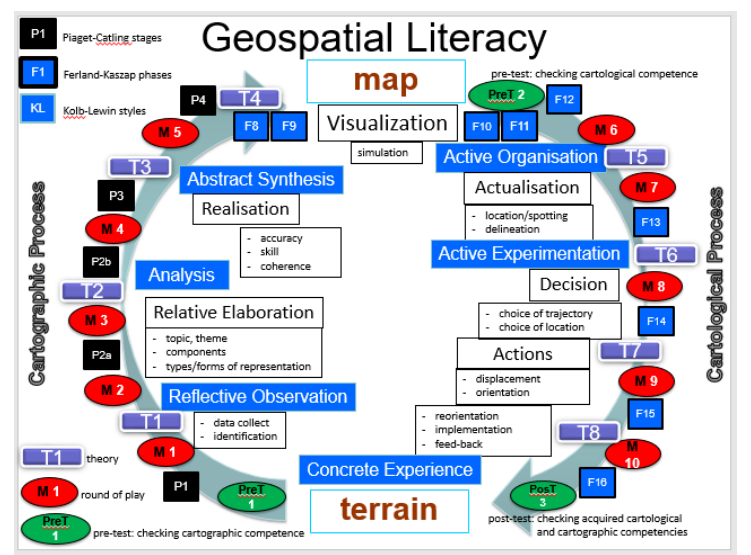

Figure 1. Geospatial Literacy Experiential Framework 